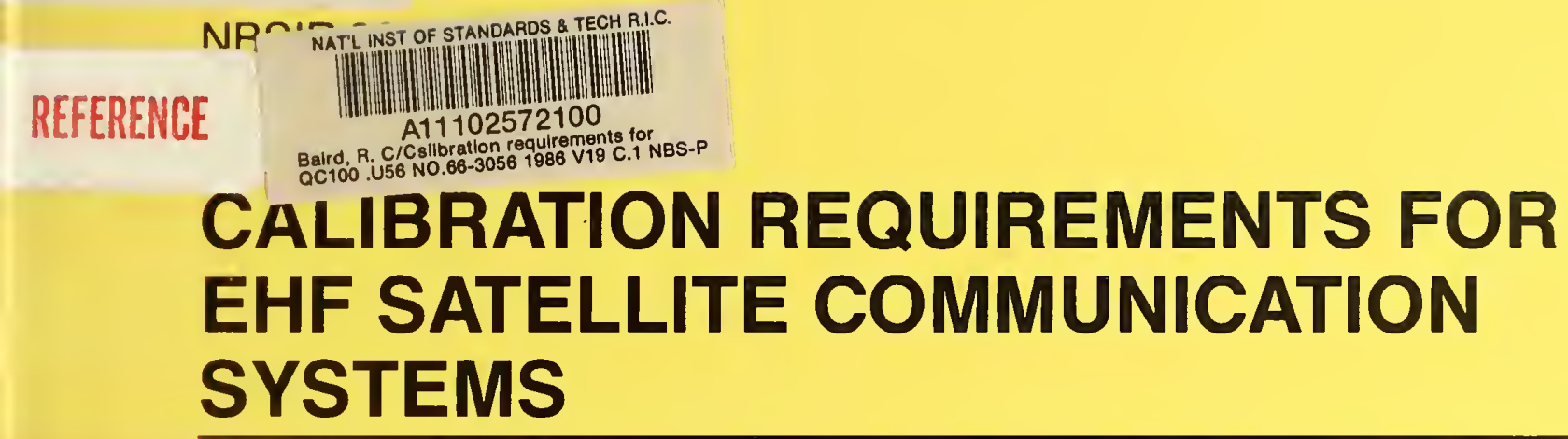

NBS

PUBLICATIONS

R.C. Baird

W.C. Daywitt

A.C. Newell

S. Perera

A.G. Repjar

D.F. Wait

A.J. Estin†

National Bureau of Standards

U.S. Department of Commerce

Boulder, Colorado 80303

†CyberLink Corporation

Boulder, Colorado 80303

October 1986

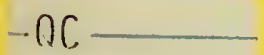

100

.1156

$86-3058$

1986 



\section{CALIBRATION REQUIREMENTS FOR EHF SATELLITE COMMUNICATION SYSTEMS}

R.C. Baird

W.C. Daywitt

A.C. Newell

S. Perera

A.G. Repjar

D.F. Wait

A.J. Estin†

Electromagnetic Fields Division

Center for Electronics and Electrical Engineering

National Engineering Laboratory

National Bureau of Standards

Boulder, Colorado 80303

†CyberLink Corporation

Boulder, Colorado 80303

October 1986

Sponsored by

Air Force Satellite Control Facility

Sunnyvale, California 94088

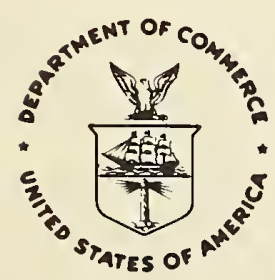

U.S. DEPARTMENT OF COMMERCE, Malcolm Baldrige, Secretary

NATIONAL BUREAU OF STANDARDS, Ernest Ambler, Director 



\section{EXECUTIVE SUMMARY}

This report concludes the present phase of an investigation by the National Bureau of Standards (NBS) into the calibration and measurement support requirements of millimeter wave (SHF and EHF) satellite systems such as MILSTAR, with particular emphasis on the role of Camp Parks Communications Annex (CPCA) as the prime Air Force Satellite Control Facility (AFSCF) common user test facility.

Following a general introduction in section 1 , sections 2 and 3 review the needs for and basis of measurements on satellite systems in terms of the link budgets, the particular role of CPCA, and the new problems that arise because of operating in the upper SHF and EHF frequency ranges. Section 4 presents an overview of the various means available for calibrating antenna gain, which is generally the key measurement that needs to be accurately accomplished.

Section 5 summarizes the calibration and measurement requirements as stated by the MILSTAR program office in the Orbital Requirements Document (ORD), Annex A (Rev. 3). Section 6 begins to develop an error budget for the measurement requirements and gives the present status of NBS measurement support capabilities. Finally, section 7 summarizes recommendations to the AFSCF, and suggests a number of tasks to assist in meeting the calibration support requirements of the EHF satellite systems.

The report points out three major areas of concern with regard to making CPCA an SHF/EHF satellite communications metrology terminal. First, without adequate methods to measure the atmospheric loss, the accuracy of effective isotropic radiated power (EIRP) measurements in the $20-45 \mathrm{GHz}$ range can be no better than 0.5-3 dB, depending on frequency and antenna elevation angle, which is inadequate for MILSTAR requirements. Second, development of required standards and measurement support services at NBS must be addressed in order for NBS to have the millimeter wave antenna gain and thermal noise measurement services needed to support CPCA. Third, if the Sun and/or Moon are used for measuring antenna gain in the millimeter region, they need to be characterized at those frequencies.

Recognizing that these concerns need to be addressed in order to establish the measurement and calibration capabilities to support AFSCF and CPCA, 
NBS has recommended a series of efforts that need to be accomplished. First, in the general area of atmospheric and noise requirements, laboratory noise standards and calibration services must be developed to support the SHF/EHF bands. Second, atmospheric losses must be accurately characterized in order to measure EIRP from a satellite. Third, the Sun and Moon must be accurately characterized at EHF frequencies if they are to be used as known sources for system calibrations. Fourth, techniques must be developed for evaluation of connectors and adaptors, since they can introduce significant errors into millimeter wave measurements.

In the general area of antenna calibration requirements, several efforts are recommended. First, NBS laboratory equipment for EHF antenna calibrations must be upgraded and supplemented. Second, modifications in near-field antenna calibration techniques that avoid cable flexing problems need to be developed. Third, swept frequency antenna gain measurement techniques need to be developed to ensure accurate gain calibrations at all operating frequencies within a band. Fourth, circularly polarized antenna gain and polarization standards and associated probes must be developed. Finally, error analyses on gain transfer calibration techniques need to be performed at SHF and EHF. 
1. Introduction...................................... 1

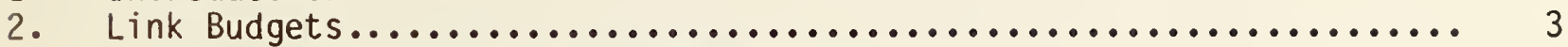

2.1 Components of a Link Budget......................... 3

2.2 Determination of System Performance.................... 5

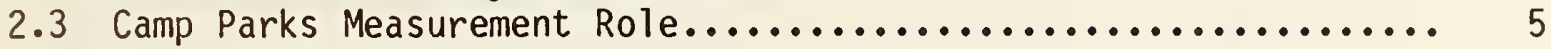

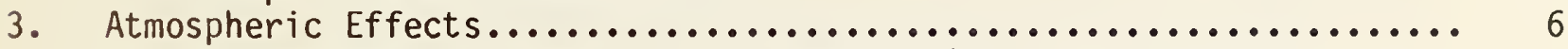

3.1 Signal Amplitude Reduction by Absorption and Scattering....... 6

3.2 Thermal Noise Increase.............................. 7

3.3 Interference Increase.............................. 7

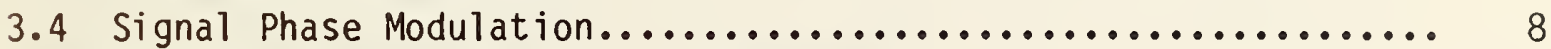

4. Techniques for Determination of Parameters................... 8

4.1 Natural Extraterrestrial Sources......................... 9

4.1.1 Gain and G/T Measurements Using Solar and Lunar Flux.... 10

4.1.2 Correction Techniques for Atmospheric Effects.......... 11

4.2 Range and Laboratory Methods for Calibrating Antenna Gain...... 13

4.2 .1 Near-field Measurement of Gain...................... 14

4.2 .2 Extrapolation Measurement of Gain.................. 15

4.2 .3 Compact Range................................ 16

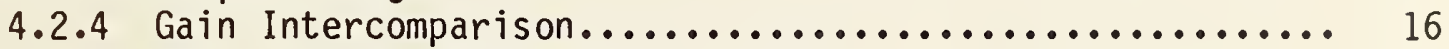

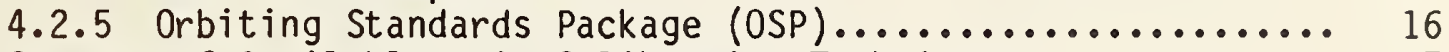

4.3 Summary of Available Gain Calibration Techniques............. 17

5. Satellite Testing/Calibration Requirements..................... 21

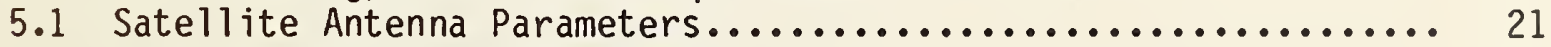

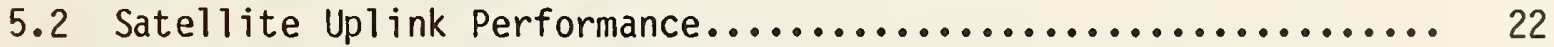

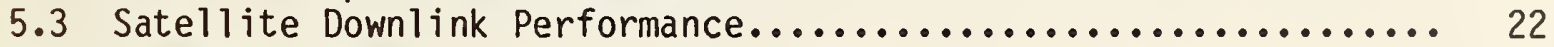

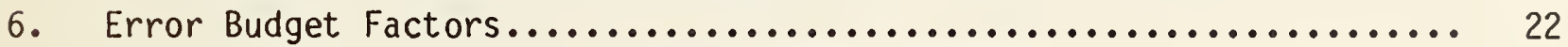

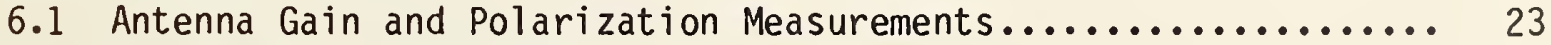

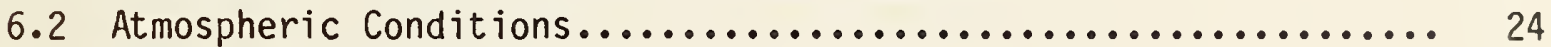

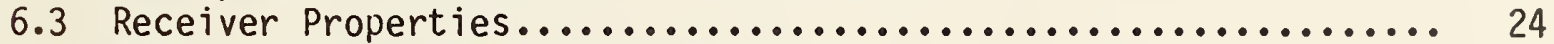

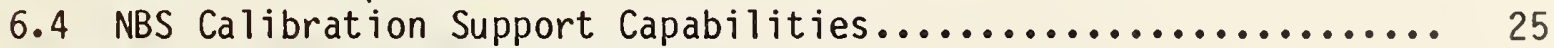

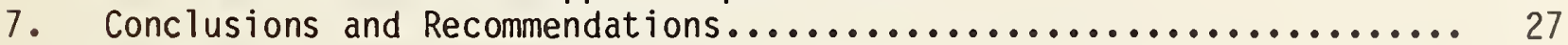

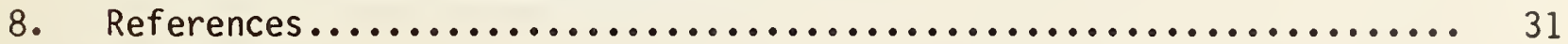



Calibration Requirements for

EHF Satellite Communication Systems

R. C. Baird, W. C. Daywitt, A. J. Estin,

A. C. Newell, S. Perera, A. G. Repjar, D. F. Wait

The calibration and measurement support requirements of millimeter wave satellite systems such as MILSTAR have been investigated. The needs for measurements on satellite systems are reviewed. An overview of the various means available for calibrating antenna gain, one of the key measurements that needs to be accurately accomplished, is presented. Essentially three new measurement problems arise because of operating in the upper SHF and EHF frequency ranges. First, without adequate methods to measure the atmospheric loss, the accuracy of effective isotropic radiated power (EIRP) measurements in the 20-45 GHz range can be no better than 0.5-3 dB (depending on frequency and antenna elevation angle), which is inadequate for MILSTAR requirements. Second, standards and measurement support services are not presently available from the National Bureau of Standards and are needed to support millimeter wave antenna gain and thermal noise measurements. Third, if the Sun and/or Moon are to be used for measuring Earth terminal G/T, Earth terminal antenna gain, or satellite EIRP in the millimeter region, they need to be appropriately characterized at those frequencies.

Key words: antenna gain calibration; atmospheric loss; effective isotropic radiated power; G/T; MILSTAR; satellite communications; solar noise flux

\section{Introduction}

The main purpose of making various types of measurements on a satellite system following deployment and during its lifetime is to obtain quantitative engineering information which enables one to anticipate performance levels. The user can thereby be assured that his requirements, as originally fulfilled by the systems designer, will continue to be met. In the event of failure or degradation of one or more elements of the system, identification of specific degraded parameters may generate an opportunity for alternate modes of usage of the system that will permit at least partial continuance of the mission of the satellite system. In addition, the agreements between the U.S. government and vendors of satellite systems, particularly satellite vendors, normally call for certain bonus payments for achieving stated levels of performance over specified periods of time. Accurate determination of performance levels therefore becomes an important factor in the procurement process as well as in the on-going operations of affected organizations. 
Broadly speaking, reliable performance measurements are required for three primary areas of satellite systems operation: a) tracking, telemetry, and command (TT\&C), which may include timing synchronization; b) baseband signal processing at the Earth sending site, in the satellite, and at the Earth receiving site, and c) transmission of radiated fields from Earth station to satellite and vice versa, including the antennas and amplifiers which generate and detect the fields. This report, as well as the National Bureau of Standards (NBS) task in support of the Camp Parks Communication Annex (CPCA), is addressed almost entirely to the last of these three areas. The scope of the report includes the identification of related measurement requirements, both those characteristic of all satellite systems and those peculiar to millimeter wave systems. In particular, applications to the 20 and $44 \mathrm{GHz}$ frequency bands are emphasized, where the former is in the SHF band and the latter in the EHF band.

The primary measure of transmission quality for digital systems is the bit error rate (or more exactly, bit error probability). For analog systems, the criterion is more complex, but in an FDM-FM system, voice channel performance can be deduced from the psophometrically weighted noise. Although these quantities can sometimes be measured directly, in general they are inferred from parameters of the link power budgets. These parameters include EIRP and gain to temperature ratio $(G / T)$ with appropriate adjustments for propagation effects. Interference, both accidental and intentional (jamming), may further complicate these measurements. Margins for environmental degradation and for system deterioration may be included as appropriate.

Following this introduction, sections 2 and 3 review the needs for and basis of measurements on satellite systems in terms of link budget parameters, the particular role of CPCA, and the new problems that arise because of operating in the upper SHF and EHF frequency ranges. Section 4 presents an overview of the means available for calibrating antenna gain, which is generally the key parameter that needs to be determined.

Section 5 summarizes the calibration and measurement requirenents as stated by the MILSTAR program office in the Orbital Requirements Document (ORD), Annex A (Rev. 3). Section 6 begins to develop an error budget for the measurement requirement and describes the immediate effects. Finally, section 7 summarizes recommendations to the Air Force Satellite Control Facility 
(AFSCF) and suggests a number of tasks to assist in meeting the calibration support requirements of the EHF satellite systems.

\section{Link Budgets}

A satellite communications channel consists of one or more pairs of links, where each pair is comprised of an uplink and a downlink. The satellite itself connects the uplink and downlink by means of a system which can vary from a simple linear frequency translator to a very complex signal processor.

The performance of a channel is measured by its carrier-power/noisedensity ratio $\left(\mathrm{C} / \mathrm{N}_{0}\right)$, and is obtained by combining the separate performances of the uplink and the downlink. Only in the case of a linear satellite transponder can the overall $\mathrm{C} / \mathrm{N}_{0}$ of the channel be obtained as a unit. Otherwise, the $\mathrm{C} / \mathrm{N}_{0}$ for each link must be obtained separately, and combined by a reciprocal addition process which is similar to calculating the equivalent of parallel resistances.

The performance of each link is predicted by its link budget, which can be derived from the radio range equation, sometimes called the Friis Transmission Formula. The link budget expression constitutes a convenient means of calculating the overall figure of merit $\left(C / N_{0}\right)$ for the link from the figures of merit of the transmitter and receiver combined with the parameters of the transmission path. The accuracy to which these elements in the link equation can be calculated, measured, or otherwise estimated, will directly affect the accuracy with which performance of the link can be anticipated.

\subsection{Components of a Link Budget}

The expression for the link budget is given as [1]

$$
C / N_{0}=E I R P+G / T-L_{\text {path }}-L_{\text {atmos }}-L_{\text {rain }}-(\text { other terms })+228.6
$$

in which all quantitites are expressed in variants of decibels.

The left side of the equation is the carrier-power/noise-density ratio, expressed in $\mathrm{dB}-\mathrm{Hz}$, which is an overall figure of merit for a digital communication link. EIRP is the product of the transmitter power and transinitting 
antenna gain in the desired direction. It constitutes a figure of merit of the transmitting station and is measured in $\mathrm{dBW}$. The figure of merit of the receiving station $(G / T)$ is the ratio of its antenna gain to the combined temperature contributions of the receiving amplifiers, receiving antenna and transmission line, and sky (including "standard sky"* and added effects of rain, clouds, etc.). Its units are $d B / K$. L Lath is the free-space loss associated with the path length and is given by

$$
L_{\text {path }}=20 \log _{10}(4 \pi d / \lambda) \text {, }
$$

where $\lambda$ is the free space wavelength and $d$ is the distance between transmitting and receiving antennas, in the same units of length. $L_{\text {atmos }}$ and $L_{\text {rain }}$ are the losses attributable to the absorption of the water vapor and oxygen in a standard atmosphere, and the increased absorption because of the precipitation along the path, respectively. At the discretion of the system designer, many other quantities can be, and frequently are, included in the "other terins." These include the effects of pointing errors, impedance mismatches, polarization mismatches, atmospheric and ionospheric depolarization, feedline losses, edge-of-coverage allowance, end-of-line allowance, stationkeeping tolerance, input and output backoff of a transponder, and other safety margins as appropriate. The 288.6 term is $-10 \log k$, where $k$ is the Boltzmann constant.

The resulting $\mathrm{C} / \mathrm{N}_{0}$ 's for uplink and downlink are then combined by means of the previously mentioned reciprocal addition process, along with other similar terms resulting from interference and jamming, intermodulation, etc. The result, $\left(\mathrm{C} / \mathrm{N}_{0}\right)_{\text {total }}$, can then be used for the composite figure of merit of the system. This assumes that the various noise and interference contributions are statistically independent of each other, which is only approximately true. A more exact evaluation requires a detailed analys is of each system.

*A standard sky corresponds to a moderately humid atmosphere $\left(7.5 \mathrm{~g} / \mathrm{m}^{3}\right.$ surface water vapor density) at latitude $45^{\circ}$ in July. No precipitation is present. 


\subsection{Determination of System Performance}

Service requirements of a satellite communications system are usually stated in terms of transmission rate $\mathrm{R}$ (bits per second) and an acceptable probability of error $P_{e}$. As a consequence of the techniques and equipment used in modulation, demodulation, coding, and decoding, the ratio of energy per bit to noise power density $\left(E_{b} / N_{0}\right)$ can be determined. With appropriate factors for coding gain, coding redundancy, and margins which may not have been included in the link budgets, the required value of $C / N_{0}$ is obtained. This is then compared with the available value, $\left(C / N_{0}\right)_{\text {total }} \cdot$ The resulting system performance levels are thereby established. If $\left(C / N_{0}\right)_{r e q}$ is greater than $\left(C / N_{0}\right)_{\text {total }}$, modification of the system is necessary.

\subsection{Camp Parks Measurement Role}

The function of the Camp Parks facility is to perform measurements to assess the performance of a satellite system or its elements. More specifically, the performance of the satellite itself is the primary focus of this activity. Thus, the CPCA does not, and presently cannot, determine the condition of various Earth terminals used with the satellite and is not an operational communications terminal itself. Of course, Camp Parks is capable of generating and receiving signals to and from the satellite, but only insofar as needed for measurement purposes. While it is desirable to be able to determine a number of characteristics of a satellite, such as saturation level of the output amplifier, gain-transfer characteristic of the transponder, gain-to-noise temperature ratio of the satellite receiver, and satellite EIRP [2], it is only the last characteristic that can be measured from the ground in an advanced nonlinear satellite such as MILSTAR. If a remote measuring capability were made an intrinsic part of the satellite and its measurement data telemetered to the Earth, then all of these characteristics could be evaluated.

In the absence of such a capability, the only quantity which can be determined is the satellite EIRP. EIRP is, however, the most crucial of these characteristics. Associated with EIRP are such quantities as antenna pattern, sidelobes, beamwidth, boresight properties, and polarization and cross polarization. These are really geographic variants of EIRP and can be measured if the satellite can be appropriately gimballed or if a mobile measuring terminal 
can be moved to appropriate spots on Earth while the satellite is held in a fixed orientation. Other boresight properties, such as polarization and cross polarization, could be determined at CPCA with appropriately designed special antennas.

Calibration of $G / T$ of operational Earth terminals is a separate task, and will not be considered here. However, calibration of the G/T of CPCA antennas is an important part of the qualification process of the CPCA mission, and such a procedure might later be adapted to measurement procedures for other Earth terminals. The use of Transportable Test Facilities is one way of accomplishing this.

\section{Atmospheric Effects}

Atmospheric effects [3] on radio propagation are almost completely negligible in the frequency range of $1-6 \mathrm{GHz}$ and have relatively little effect up to $10 \mathrm{GHz}$. Above that region, however, atmospheric modification of transmitted signals becomes increasingly important. Near and above $60 \mathrm{GHz}$, atmospheric effects on a satellite/Earthlink utterly dominate all other factors. These effects are attributed to two separate phenomena: molecular absorption by water vapor and oxygen, and absorption and scattering by discrete particles consisting principally of water droplets. The latter phenomenon introduces the most difficult problems in operating a satellite link or in accurately measuring the performance of this link. This is because the effect of water droplets is not only more profound but also more widely variable from time to time and from place to place and depends on size, shape, and distribution parameters of the droplets.

\subsection{Signal Amplitude Reduction by Absorption and Scattering}

The most direct atmospheric effect on the link is a reduction in received signal level that can be divided into 1) an invariant sky effect due to the absorption of the "standard" sky (described in section 2.1) which is primarily a function of frequency, and 2) absorption resulting from rain. Prediction of the former can be accomplished by standard techniques to within several tenths of a decibel up to about $20 \mathrm{GHz}$, and to within a decibel or so between 20 and $45 \mathrm{GHz}$. In this way, both the performance of the link and relevant parameters such as EIRP of the satellite can be fairly accurately established. Under 
conditions of visible heavy clouds and rain, however, establishing these parameters is much more difficult and less accurate. Calculations from atmospheric models may not provide the desired accuracies. Thus, either a direct measurement technique may need to be developed and used, or a more accurate way of determining these important atmospheric pararneters inust be established. The loss in signal level under severe conditions can be $20 \mathrm{~dB}$ or more at $44 \mathrm{GHz}$, and therefore can nearly obliterate the signal and can completely swamp out its measurement accuracy.

\subsection{Thermal Noise Increase}

One effect of atmospheric attenuation is to decrease the signal level received. Further, because the atmospheric temperature is much higher than the galactic background temperature, atmospheric absorption has the added detrimental effect of increasing the noise temperature at the receiver. In other words, the $\mathrm{C} / \mathrm{N}_{0}$ ratio at the Earth receiving terminal is degraded both by decrease in signal level and by an increase in operating noise temperature.

\subsection{Interference Increase}

Another adverse effect of the atmosphere on signals above $10 \mathrm{GHz}$ is that interference from other sources, which are nominally isolated by beam directivity and by orthogonal polarizations, may in fact be redirected into the signal channel in question. Water droplets in particular have the relatively strong effects of defocusing a radio wave and of changing its polarization. A military system such as MILSTAR is designed to be relatively impervious to intentional jamming. It is therefore also relatively insensitive to added interference which may be redirected into its receiving antennas because of atmospheric scattering. Moreover, MILSTAR does not use dual polarization, and the small amount of power scattered into or out of the desired polarization is of minor importance. This phenomenon may therefore be put to beneficial use. Since the orthogonal polarization mode is not used, it may be possible to monitor it and, by measuring the amount of signal scattered into that cross polarization, to obtain an estimate of the absorptive attenuation in the copolarization channel. 


\subsection{Signal Phase Modulation}

Phase variations in the transmitted signal can be attributed to two separate effects occurring in the atmosphere $[4,5]$. Phase delay fluctuations take place because a wave passes through a medium of variable refractivity. These phase fluctuations increase linearly with frequency, but are diminished as the physical antenna diameter approaches the scale factor of fluctuations, which is normally about $30 \mathrm{~m}$. Between 20 and $44 \mathrm{GHz}$, the phase fluctuations can be of the order of several hundred degrees for an antenna which is one or two meters in diameter.

A second type of phase variation occurs because of wavefront ripple caused by a "lumpy" medium. These variations in phase are rapid, and are called phase scintillations. They are proportional to the square of frequency and to antenna size, and decrease with increasing angle of elevation. For high elevation angles, these phase variations are of the order of ten degrees, which may have an effect on very high data rate systems. Another effect is an apparent reduction in antenna gain of as much as one decibel.

\section{Techniques for Determination of Parameters}

In order to have a complete understanding of any radio link, three quantities must be established: 1) the properties of the transmitting station; 2) the properties of the transmission medium, and 3 ) the properties of the receiving station. If any two of these are known, one additional measurement will determine the third. Conversely, if two or more are not known, a single measurement cannot evaluate the properties in an unambiguous way. The general procedure will be, in the present requirement, to calibrate the last of these quantities by independent means, to at least approximate the second, possibly in real time, and therefore to measure the first at the (Earth) receiver. This section will address various techniques of calibrating the receiving station.

The receiving station calibration involves a combination of a voltage or power measurement, usually through a high gain amplifier, and an antenna gain measurement. Other properties, such as receiver noise level and antenna cross polarization, sidelobes, etc., do not enter directly into the measurement, but do serve to limit the accuracy that can be achieved. 
The most important quantity required in the calibration of the receiving station is the gain of the receiving antenna. Available techniques for making this calibration include the use of extraterrestrial radiation sources (whose spectral emissions have been reliably measured) as well as several different laboratory techniques. The remainder of this section will be devoted to an examination of these techniques, with summaries of advantages, disadvantages, and quantitative estimates of the accuracies available with each method.

\subsection{Natural Extraterrestrial Sources}

Several celestial bodies have been used or proposed for use as sources of known flux levels to calibrate Earth stations [6]. These include such radio stars as Cassiopeia A, Cygnus A, Taurus A, Orion A, and Virgo A, plus bodies from within our own solar system such as the Sun, the Earth's moon, and certain planets.

Below about $10 \mathrm{GHz}$, the fluxes received on Earth from Cas $A$ and Cyg $A$ are the strongest, and are predictable to an absolute accuracy of between 5 and 10 percent. Above 10-15 GHz, Tau A and Orion A become the dominant stellar sources, but even they are comparatively weak (below 500 flux units or F.U. where one F.U. $=10^{-26} \mathrm{~W} / \mathrm{m}^{2} / \mathrm{Hz}$ ) and their intensities have not been well established. The Sun has long been attractive because it is the strongest natural source available above $10 \mathrm{GHz}$, with nearly 6,000,000 F.U. at $15 \mathrm{GHz}$ and increasing with frequency. There are, however, two restrictions on the use of the Sun. It is close enough to us that it subtends about 0.5 degrees of arc and hence its nonuniformity will be resolved by high gain antennas. It is extremely active and therefore its radio emission varies with time, 10cation on the solar disk, and frequency. Despite these handicaps, this is a potentially useful calibrating source under some circumstances. The Moon is a source of comparable angular extent. It has much lower intensity but greater uniformity. Planets are far enough away to constitute essentially point sources and may (especially Venus) be bright enough to be useful in the SHF/EHF frequency range. The disadvantage of planetary sources is that, depending on the relative orbital location with respect to the Earth, the distance and therefore the received flux varies greatly at different times of the year. 


\subsubsection{Gain and G/T Measurements Using Solar and Lunar Flux}

If the flux from a celestial source such as the Sun or Moon is known, then it can be used as a reference signal to calibrate satellite EIRP in a comparison measurement. Earth station antenna gain can be determined by measuring the absolute power out of the antenna when the receiving system is "looking" at the reference source; and the receiving system G/T can be determined $[7,8]$ by measuring the relative power out of the antenna when the system is pointed at the cold sky background.

Above $5 \mathrm{GHz}$, the Sun and the Moon are the only celestial sources that are useful if the receiving system has a "sma11" antenna (less than $55 \mathrm{~dB}$ gain). Below $5 \mathrm{GHz}$, the flux from the Sun varies greatly over short time spans, so only the moon can be used unless the frequency is low enough (about $3 \mathrm{GHz}$ for $55 \mathrm{~dB}$ gain systems) that radio stars such as Cassiopeia $A$ are sufficiently intense.

For the Moon, a minimum antenna gain of about $40 \mathrm{~dB}$ is needed to produce an antenna temperature (signal-to-noise ratio) great enough for measurement purposes. The maximum antenna gain is about $55 \mathrm{~dB}$ and is determined by the largest antenna for which the correction for the finite size of the source (called the "star shape correction factor") can be calculated accurately. So the Moon is potentially useful as a source for systems with antenna gains between 40 to $55 \mathrm{~dB}$ and for frequencies below about $45 \mathrm{GHz}$ that transmit through the atmosphere.

The Sun is a more intense source, and antenna gains as low as 13 dB near $1 \mathrm{GHz}$, or $29 \mathrm{~dB}$ near $45 \mathrm{GHz}$ give usable outputs. The maximum antenna gain is again set by the accuracy of the star shape correction factor and is about $49 \mathrm{~dB}$.

Thus, in principle, one can use the Sun or Moon as a reference source for measuring Earth terminal G/T and satellite EIRP at frequencies below about 45 $\mathrm{GHz}$. The measurement system requires an antenna with gain between about 29 and $55 \mathrm{~dB}$ which can be pointed to the Sun or Moon.

However, before the Sun and Moon can be used as reference sources above $10 \mathrm{GHz}$, several problems need to be resolved. Above $10 \mathrm{GHz}$, the theoretical models for the flux as a function of frequency and "star shape" correction factors for the Moon need to be developed. Also, as the frequency increases, 
the atmospheric effects become more pronounced. Near $20 \mathrm{GHz}$, water vapor significantly attenuates electromagnetic propagation so that one can expect the atmospheric absorption to vary significantly if the water vapor profile of the atmosphere changes during the measurement. Consequently more careful and more frequent measurements of atmospheric loss are needed than at the lower frequencies.

Another problem that needs attention at the higher frequencies is the effects of rain and clouds on the measurement accuracy. It will be impractical to always make measurements of EIRP on cloudless days.

\subsubsection{Correction Techniques for Atmospheric Effects}

The atmosphere absorbs and scatters radiation traveling through it, thus reducing the magnitude of and altering the polarization of a received signal. In clear weather, oxygen and water vapor are the significant absorbers, with variations in the absorption resulting primarily from changes in the water vapor density. The temperature and pressure profiles usually vary little from hour to hour and day to day within the same air mass but change significantly across atmospheric weather fronts. These changes occur on a large scale spanning hundreds of kilometers, mainly affecting the troposphere, with smaller scale variations of importance near the surface. Diurnal changes in temperature occur from surface heating and cooling.

The water vapor concentration in the lower troposphere is highly variable, especially within 1 or $2 \mathrm{~km}$ of the surface, and occurs both on a large weather mass scale and on the smaller scale associated with individual clouds. The air within a cloud is generally saturated, while outside lower humidity values are usually observed.

Depolarization caused by rain attenuates the signal by both scattering the radiation out of the beam and rotating the linear polarization components. Thus, in experiments where the orthogonal polarization is also used, crosstalk occurs. Although the rain attenuation is not directly measurable, it can be determined by measuring the amount of rain-induced cross polarization of the received signal, given some knowledge of the drop-size distribution and rainfall rate. 
The zenith attenuation due to oxygen and water vapor absorption varies from about $0.05 \mathrm{~dB}$ at $10 \mathrm{GHz}$ to over $100 \mathrm{~dB}$ at $60 \mathrm{GHz}$ for a water vapor density of $10 \mathrm{~g} / \mathrm{m}^{3}$ which corresponds to a dew point of $+10^{\circ} \mathrm{C}$. The sky emission associated with this oxygen and water vapor absorption varies in brightness temperature from 5 to $300 \mathrm{~K}$ over this same frequency range, and competes with the desired signal reception.

A11 of these effects can be estimated with more or less accuracy depending on the state of the atmosphere at the time. Algorithms exist for determining atmospheric loss and emission even in inclement weather. These algorithms should be used when measurements must be done in bad weather, but for really accurate measurements clear, stable weather is necessary. The algorithms still need to be examined and a rough estimate of their accuracy determined. The discussion that follows, therefore, assumes good weather.

As far as atmospheric conditions are concerned, a requirement for accurate gain and $G / T$ measurement using an extraterrestrial source is the ability to point away from the source and still encounter the same atmospheric path loss and radio emission. This roughly defines what is meant in this report by the phrase "clear, stable weather," even though some variable cloud cover with a small amount of attenuation may be present. Measuring the atmospheric loss at various elevation angles for two different azimuth angles will produce the same results (within the statistical fluctuations of the measurement) if the atmosphere is suitable in the above sense. Conditions are sufficiently stable if the loss measured before and after the gain or G/T measurements are the same.

Above $10 \mathrm{GHz}$ the atmospheric loss increases, and the magnitude of the loss must be determined more accurately in order to estimate transmission effects with the same accuracy as for lower frequencies. Thus, while calculation of the loss using only surface data suffices below $10 \mathrm{GHz}$, a more elaborate procedure is required in the higher frequency ranges. Two direct methods for measuring the loss that do not require a priori knowledge of the ground station antenna gain are available, the "extinction" and the "tipping curve" (atmospheric emission) methods [9].

In the extinction method, the relative flux from an extraterrestrial source (e.g., the Sun) is monitored from zenith to a low elevation angle. When the logarithm of the source's relative strength, as measured at the 
ground station, is graphed as a function of the cosecant of the elevation angle, the slope of the resulting straight-line fit to the points is the zenith atmospheric loss. This is a slow process, requiring at least five hours to complete, because the source must travel across a large sky angle. In addition, the weather may change during this process, thus degrading its accuracy. The tipping curve method is similar except that the radio emission of the sky is used instead of an extraterrestrial source. For the present application, the latter method is preferable since it can be performed at any time of day or night and at any azimuth angle. When used in conjunction with the Earth terminal measurement system (ETMS) on an antenna with an $800 \mathrm{~K}$ (or smaller) system noise temperature, the tipping method appears to work up to frequencies where the zenith loss does not exceed $1 \mathrm{~dB}(40-50 \mathrm{GHz})$. Final evaluation of this method's feasibility and overall accuracy will require experimental tests not yet performed. The details and highlights of this method are presented in [8], an NBS report on work accomplished as part of this EHF study.

In order to determine the loss above the $1 \mathrm{~dB}$ limit just mentioned (or as a backup if the tipping curve/ETMS approach proves unworkable), a dual frequency radiometric technique is available that determines the water vapor content of the atmosphere by performing measurements at $22.2 \mathrm{GHz}$ and $31.65 \mathrm{GHz}$. The result is used to calculate the loss at frequencies above the $1 \mathrm{~dB}$ limit. Instrumentation for this technique requires a considerable outlay in time and manpower to construct but may be the best solution in the long run. Error estimates remain to be determined.

\subsection{Range and Laboratory Methods for Calibrating Antenna Gain}

The period before and during World War II was marked by enormous steps in the development of techniques for measuring the characteristics of antennas. Until the advent of the space age in the early 1960s, however, these advances were characterized by refinements in accuracy and convenience, rather than by conceptual changes. Pattern integrators, positioners, and automatically controlled signal sources and receivers facilitated data acquisition and presentation, but did not improve the intrinsic quality of the data, nor did they extend the techniques to electrically (and physically) large aperture 
antennas then coming into use for space communications. The critical requirements of space communication systems pushed antenna and component designs to higher performance levels and reduced the margins available for design and measurement tolerance. As a result, fundamental changes took place in antenna measurements. Conventional far-field ranges were forced to yield to impossible requirements. For example, to remain in the far field a 20-meter antenna operating at $\mathrm{C}$-band would require a separation from its test probe of at least $10 \mathrm{~km}$. Even a 2-meter antenna at $44 \mathrm{GHz}$ must be over $1 \mathrm{~km}$ from its probe. The combination of more stringent accuracy requirements, more severe multipath errors, and the high cost of acquiring and maintaining such large ranges has relegated conventional far-field ranges to a position of reduced usefulness. Accordingly, new approaches were devised for antenna measurements, including compact ranges, near-field probing techniques, extrapolation ranges, tapered anechoic chambers, and indirect measurement techniques. Thus the antenna measurements problem has grown to equal complexity with the antenna design problem and must be given comparable weight by the antenna engineer.

In this section, we will address methods of gain determination available for calibration of Camp Park facilities.

\subsubsection{Near-Field Measurement of Gain}

Near-field measurements of an antenna $[10,11,12]$ are obtained by careful sampling of the phase and amplitude of the aperture field in sufficiently fine detail that any desired radiation property of the antenna can be deduced. By means of an appropriate Fourier transformation, the aggregate near-field data are used to calculate the far-field amplitude and polarization in almost any desired direction. If a calibrated probe is used to acquire the data, then the field amplitudes can be referred to an absolute level. This yields the gain of the antenna in the boresight direction and also detailed pattern information. The measurement process is very accurate, but can be complex and expensive for large antennas; if only boresight gain and polarization are needed, other procedures may be preferred.

A thorough error analysis procedure has been developed for this process. A gain uncertainty as small as two tenths of a decibel is routinely attainable. A more exact evaluation of uncertainty may be achieved, depending 
on the specific frequencies and properties of the antenna being measured. Several problems become more severe for millimeter waves: 1) mechanical misalignments and instabilities are a larger fraction of the wavelength and therefore phase errors become more important; 2) flexing of cables required during movement of the probe carriage can introduce disproportionately high phase errors in the millimeter spectrum, and 3) connectors, both waveguide and coaxial, must be precisely made. It is currently difficult to obtain connectors that provide repeatable connections.

\subsubsection{Extrapolation Measurement of Gain}

The extrapolation measurement $[13,14,15]$ is specifically designed for determining the on-axis (boresight) gain and polarization parameters. NBS has developed a rigorous means for determining the far-field gain products of two antennas which are located in each other's near fields. If three antennas are permuted in pairs through such measurements, the gain of each antenna can be separated out. On an extrapolation range, this process is conducted in the near-field zone of the antennas, and then the effects of proximity and multiple reflections are rigorously removed. The axial ratio, tilt angle, and sense of polarization for each antenna can also be determined by measuring the amplitude and phase changes which occur when one antenna (of each pair) is rotated about its boresight axis.

This measurement technique is not as sensitive to mechanical misalignments and instabilities as the near-field scanning method described in section 4.2.1. Further, movement of the antennas with respect to each other occurs only in one dimension, rather than two. Therefore, rails and trolleys can be made more rigid, thus keeping positional uncertainties to smaller values. As a result, the extrapolation method does not have a readily discernible upper frequency limit and should be quite useful for evaluating millimeter wave standard antennas. The most severe constraint is that the available extrapolation ranges are somewhat limited in the weight and physical size of antennas that can be handled. Also, the length of an extrapolation range must be sufficient to permit the separation distance between the antennas to vary from about one-fourth to one-half $D^{2} / \lambda$ at the minimum to about $2 D^{2 / \lambda}$ at the maximum. 


\subsubsection{Compact Range}

The compact range $[16,17]$ simulates a far-field range by $i 11$ uminating the antenna under test with a collimated beam, while surrounding the entire assembly with absorbent material. The collimated beam is formed by illuminating a precision paraboloidal surface with a point source antenna located at the focus of the paraboloid. This beam can be assumed to have a plane wave configuration in the central 6-10 percent of its cross section. Calculations indicate that a surface roughness tolerance of $0.007 \lambda$ in the reflector can result in $0.5 \mathrm{~dB}$ amplitude error. Other effects such as scattering from the reflector edges and stray radiation from the point source further degrade the results obtained by this technique. Absolute gain measurements with a compact range are not feasible. Comparative gain measurements are possible but their accuracy limitations have not been analyzed.

\subsubsection{Gain Intercomparison}

If an antenna which has been previously calibrated is available, a gain comparison technique can be used to determine the gain of an unknown antenna by simultaneously comparing the signals received from a satellite (or celestial body) by both antennas [18]. If the antennas are co-1ocated, this has the further advantage of eliminating atmospheric effects since both received signals will have traversed paths having essentially identical characteristics.

The gain intercomparison technique requires a relatively small amount of special instrumentation; however, one must compensate for various possible sources of error. If the gains of the reference antenna and the antenna under test differ by more than about $30 \mathrm{~dB}$, a significant source of error can be introduced into this technique. It is not expected that this problem will be encountered with the antennas under consideration for MILSTAR Earth terminals. This technique will become more important at higher frequencies and may be the only reliable and practical method of calibrating the larger Earth stations.

\subsubsection{Orbiting Standards Package (OSP)}

Near-field scanning techniques require elaborate instrumentation and personnel with expertise in near-field theory and measurements. Conceivably, it is possible to develop transportable near-field facilities to perform 
measurements at a field site, but the process would be expensive and difficult. Consequently, a new concept, the Orbiting Standards Package (OSP) was investigated at NBS toward the late 1970s for use in conjunction with field measurements as well as routine antenna testing at manufacturer's facilities. As was stated in section 4.2, far-field measurements of an electrically large antenna on a terrestrial range require extremely large separations which introduce major problems of undesirable ground reflections. However, if the remote terminal of such a range could be located on a satellite, the requirements of separation and multipath elimination could both be met. Developments in metrology over the previous decade now make practicable the tasks of remote measurernent, control, and recalibration. The advantages of the OSP are: 1) both uplink and downlink measurements can be made without loss of accuracy caused by transference; 2) atmospheric effects can be directly compensated; and 3) testing of the system can be designed so as to exercise it under many configurations. It does mean, however, that the OSP must be designed into a satellite.

\subsection{Summary of Available Gain Calibration Techniques}

The various regions of applicability of available techniques of calibrating antenna.gain are shown in figures 1 and 2. The three most important parameters of an aperture antenna, any two of which are independent, are its frequency, physical diameter, and ratio of diameter to wavelength. These parameters also serve to set limits on reasonable configurations of such antennas. On the frequency scale, the bounds of this region are approximately $200 \mathrm{MHz}$ to $75 \mathrm{GHz}$. Physical size limits the top of the region to well under 100-m diameter. Electrical diameter (diameter expressed in wavelengths or $D / \lambda$ ) cannot be less than about one-half and rarely is higher than about 500 because of the limitations on surface precision of the reflector and the difficulty in pointing a beam which is sharper than about 0.1 degree. These are not theoretical limitations, but merely represent common practical bounds. These constraints define the region in which aperture-type antennas are found and the heavy line delineates this region. 




Figure 1. Regions of applicability of range-type antenna gain measurements. 
The usefulness of these charts is that, for a given antenna size and frequency range, one can identify feasible methods of gain calibration. The techniques which are included in this chart are those methods of primary calibration that have been discussed in section 4 of this report. They do not include: gain comparison, because that is a secondary calibration; OSP, because the concept has not been implemented and tested; or conventional farfield ranges, because their usefulness depends on the terrain and height of towers available.

Near-field forms of measurement are constrained to basically triangular regions on this chart. The right side of the triangle results from a limit in phase uncertainty defined by mechanical precision of the trolleys and tracks used to move the antenna under test or the test probe. The upper side of the triangle is determined by the largest antenna the range is capable of handling. The diagonal side of the triangle is limited by the sidelobe radiation from the antenna. At small values of $D / \lambda$, the sidelobe radiation increases enough that errors caused by multipath effects begin to dominate and degrade accuracy. Notice that with these near-field measurement techniques, a large value of $D / \lambda$ is not in itself a limiting factor.

Calibration by natural extraterrestrial sources is shown in figure 2. Stellar flux measurements are limited in the range of frequencies between 1 and $15 \mathrm{GHz}$. At the lower frequency end, nonthermal galactic radiation begins to dominate the sky and interferes with specific stellar sources. At the upper end, stellar flux levels become too weak for reasonable accuracy. The electrical size of the antenna is significant because too large an antenna has a beamwidth smaller than any available star and, therefore, resolves the structure of the star. Too small an antenna does not have a sufficiently high $G / T$ ratio to detect the star. A variability of $D / \lambda$ from about 30 to 500 can be successfully handled with Cas $A$.

The solar disc is a very bright source which approaches blackbody radiation characteristics in the millimeter range. Its frequency limitations are from $5 \mathrm{GHz}$, below which highly variable coronal radiation dominates, to about $45 \mathrm{GHz}$, where atmospheric uncertainties become unacceptably high. Acceptable values of $D / \lambda$ vary between 20 , below which sidelobe levels are excessive, and 150, above which the main beam resolves details of the solar disc. 


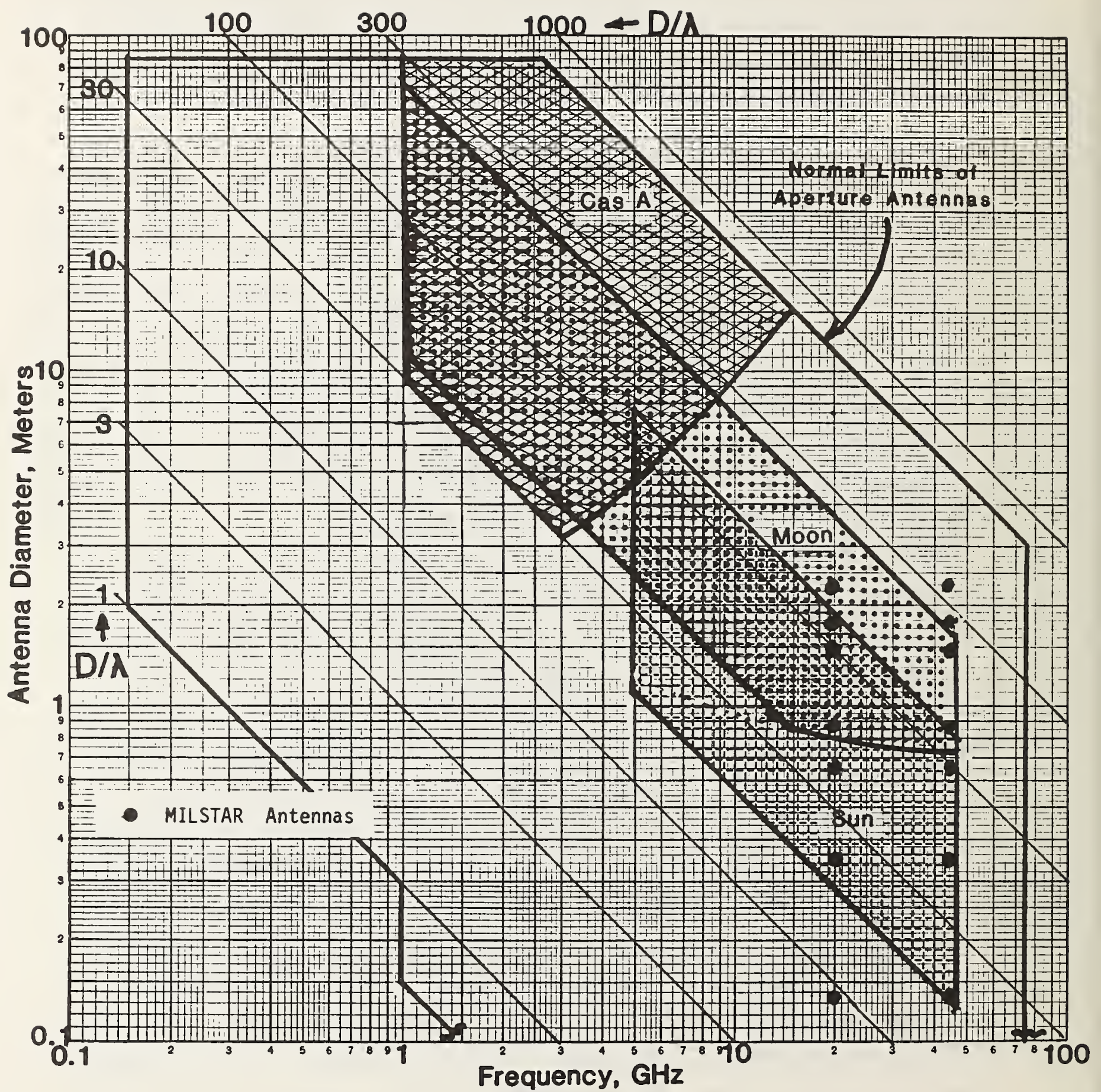

Figure 2. Regions of applicability of celestial-type antenna-gain measurements. 
The Earth's moon, although having weaker radiation than the Sun, is useful over a wider frequency range, from 1 to about $45 \mathrm{GHz}$. The minimum antenna $D / \lambda$ is about the same as for the Sun because of the sidelobe problem, but the maximum $D / \lambda$ increases to about 200 because some resolution of the more uniform lunar disc is acceptable. However, because of the weaker radiation from the Moon, systems with high noise temperatures may find the Moon less useful than the Sun. Both Cas $A$ and the Moon encounter a $Y$-factor limit which is a function of the achievable receiver temperature at the operating frequency, the gain of the antenna, and the brightness of the source. This limit accounts for the curved boundary at the lower edge of these regions.

Approximately seven different Earth station antennas are planned for use with MILSTAR. These range in size from 0.15 to $2.30 \mathrm{~m}$. These antennas have been indicated on figures 1 and 2 for both uplink and downlink use.

\section{Satellite Testing/Calibration Requirements}

This section summarizes the relevant on-orbit testing and calibration requirements as delineated in the MILSTAR ORD, Annex A, Rev. 3. These requirements are to be imposed on the AFSCF CPCA and other related units. The primary function of the requirements is to provide initial on-orbit calibration of communication payloads. The second function is to support anomaly testing as directed by the Satellite Test Center (STC). The third function is to support any additional satellite test efforts undertaken by the STC. The requirements fall into three categories: satellite antenna parameters, satellite uplink performance, and satellite downlink performance.

\subsection{Satellite Antenna Parameters}

The satellite transmit and receive antennas operate in circular polarization, and have a number of steerable and fixed beams available. The requirement for polarization measurement is stated in terms of axial ratio, with tilt angle and polarization sense being unneccessary. (It is quite logical to ignore these last two polarization parameters, for sense is either "right" or "wrong," and tilt angle becomes indeterminate in the limit of perfect circular polarization.) Other antenna characteristics such as beamwidth, beam pointing, and antenna coverage, as was mentioned earlier in this report, 
can be determined by using test equipment based on an equivalent of CPCA capability in a mobile land or airborne configuration.

\subsection{Satellite Uplink Performance}

The basic requirement of uplink performance is to obtain BER information at the receiving Earth terminal ( $C P C A$ ) while the satellite is illuminated with different Received Isotropic Power (RIP)* levels. This requirement is specified in terms of uncertainties of the RIP which depend on the particular hopping configuration of the uplink. In particular, the allowable uncertainty for normal hopping is $\pm 0.5 \mathrm{~dB}$; for partial band measurement it is $\pm 0.3 \mathrm{~dB}$; and for TRANSEC zero** mode, $\pm 0.2 \mathrm{~dB}$. At present, the on ly means of complying with this requirement is with a single channel power meter of unknown characteristics which is supplied by the satellite vendor.

A satellite G/T parameter is also desirable, but specific requirements are not stated in the ORD.

\subsection{Satellite Downlink Performance}

Satellite downlink performance is specified in terms of its EIRP for various beams. This requirement is also stated for the same three modes of hopping operation mentioned in the previous paragraph: $\pm 0.5 \mathrm{~dB}, \pm 0.3 \mathrm{~dB}$, and $\pm 0.2 \mathrm{~dB}$, respectively.

\section{Error Budget Factors}

In this section we shall convert the requirements of section 5 into measurement system specifications. As a first approximation, we assume that the variances of the different elements in the measurement are statistically independent and therefore are additive. This is equivalent to stating that the uncertainties combine by the root-sum-quare process. The $0.2 \mathrm{~dB}$ overa11 uncertainty requirement identified in section 5 suggests a goal of $0.1 \mathrm{~dB}$ for the measurement uncertainty of each element. The antenna and propagation requirements are essentially independent of the mode of hopping. For this

\footnotetext{
*Flux density at the satellite times $\lambda^{2} / 4 \pi$, where $\lambda$ is the wavelength; or equivalently, the power received by an isotropic antenna.

**TRANSEC zero is a stop-hop mode, used for default.
} 
reason, the tolerances on those elements must be tied to the tightest of the requirements, $\pm 0.2 \mathrm{~dB}$. The associated terminal equipment, receivers, and power meters will have their own intrinsic performance limitations. This is particularly true of the satellite receiving terminal, where the characteristics of the equipment are not under the control of AFSCF.

Basic measurement support capabilities available from NBS will be addressed as appropriate.

\subsection{Antenna Gain and Polarization Measurements}

A gain uncertainty requirement of $\pm 0.2 \mathrm{~dB}$ on the associated test antennas places their calibration at the limit of the present state of the art, which still may not be adequate to satisfy other error criteria. For example, in the on-board power detection system, the error in the uplink received isotropic power measurement is bounded by $\pm 0.2 \mathrm{~dB}$. This will require antenna gain accuracies well within $\pm 0.15 \mathrm{~dB}$ since error uncertainties in attenuators, reflection coefficients, connectors, etc. are also contributing factors to the total error. In light of this, error budgets need to be constructed that reflect a more realistic understanding of the specific measurement involved.

A second requirement is to measure the transmit and receive axial ratios of the satellite antenna to an uncertainty of $0.2 \mathrm{~dB}$. Assuming that the axial ratio of the antenna under test is about $0.5 \mathrm{~dB}$ (or equivalently its cross component is $30 \mathrm{~dB}$ below its main component), one would require the measuring antenna to have an axial ratio in the order of $0.2 \mathrm{~dB}$ if circularly polarized probes were utilized. A linear probe could be rotated for the axial ratio measurement, but in either case depolarization due to atmospheric effects needs to be understood for an accurate error budget determination.

These requirements impose additional conditions on the quality of the measuring antenna, as well as on the accuracy of its calibration. Under these circumstances, it may prove necessary to design and build a special measurement antenna, rather than use one of the standard Earth station units being procured for operational use. Once such an antenna is obtained, however, the calibration of its axial ratio can be accomplished in conjunction with the gain calibration by the extrapolation technique described in section 4.2.2. 


\subsection{Atmospheric Conditions}

With a goal of limiting the atmospheric contribution to the error budget to $\pm 0.1 \mathrm{~dB}$, very stringent controls must be exerted on the meteorological conditions under which measurements are made. If clear air conditions are present, zenith attenuation from sea level is about $0.3 \mathrm{~dB}$ at $20 \mathrm{GHz}$ and about $0.5 \mathrm{~dB}$ at $44 \mathrm{GHz}$. The standard deviation of these quantities over all seasons and at many locations is several tenths of a decibel, but can be considerably reduced by using additive corrections for local water vapor density and temperature at the surface. In addition, a geometrical correction factor must be used for angle of elevation.

The atmospheric corrections for cloudy or rainy conditions are much larger, and are far from accurate enough to satisfy the goal of an atmospheric correction to $\pm 0.1 \mathrm{~dB}$. Therefore, measurements must be strictly limited to clear sky conditions, or a new and involved means of correcting for clouds and rain must be developed and used.

\subsection{Receiver Properties}

In evaluating received power levels, either at the Earth terminal or at the satellite, measurements are made of either the IF or video detected power which has been processed by the receiver, converters, and amplifiers. The Earth Terminal Measurement System (ETMS) designed by NBS specifically compensates for receiver imperfections and thereby makes a power measurement which is accurately related to the power received at the antenna. If other types of power measurements are used, especially at the satellite, receiver properties such as linearity and dynamic range, short- and long-term gain stability, variation of the detection law, and possibly VSWR must be known for a reliable error evaluation. For the hopping and partial band measurement modes, the receiver response time is also needed. The test-signal/noise-temperature ratio and receiver bandpass characteristics affect the accuracy of measurement. These quantities may also require corrections that depend on the particular hopping pattern.

Particularly at the satellite, there may be significant drifting in the receiving and power measuring systems because of aging, environmental conditions, or the stresses of launching. For this reason, the NBS OSP would 
include remote self calibration procedures so as to retain confidence in the accuracy throughout the life of the satellite.

\subsection{NBS Calibration Support Capabilities}

NBS measurement services now provide only spotty coverage of parameters of interest to the AFSCF in the EHF region. Table 1 is a summary of the available services for power, attenuation, reflection coefficient, antenna characteristics (gain, pattern and polarization), and thermal noise sources. It is apparent from the table that there are several significant gaps in services in the MILSTAR bands. The table also includes the priority recommendations of the Promote National Microwave Standards (PNMS) Committee. This is an IEEE sponsored group, independent of NBS, composed of representatives from major U.S. industries who have identified industry needs and lack of NBS capabilities. One of the PNMS committee goals is to make people aware of the importance of and need for NBS standards and metrology support throughout the active microwave and millimeter wave frequency bands. The committee's highest priority is on the development of measurment support services in the 18 to $50 \mathrm{GHz}$ bands.

The tri-services Calibrations Coordination Group (CCG) of DoD is another organization which is concerned with obtaining adequate metrology support from NBS. This group is responsible for identifying calibration requirements throughout DOD and then making sure that the needed measurement capabilities exist in DoD calibration labs. Since DoD calibrations must be traceable to NBS, CCG works closely with NBS and encourages the timely development of measurement services required to support new and existing military systems. A major concern of CCG's is the lack of NBS standards and metrology capability in the 18 to $60 \mathrm{GHz}$ bands needed to support the MILSTAR program.

Therefore, given specific (and compatible) guidelines by both the private and military sectors, NBS is directing its efforts into developing the most needed services, as resources allow. AFSCF needs are (fortunately) on that path of development. However, the time frame within which the needed EHF standards and measurement support services will be established depends upon the total level of support NBS receives for the development of microwave metrology. 


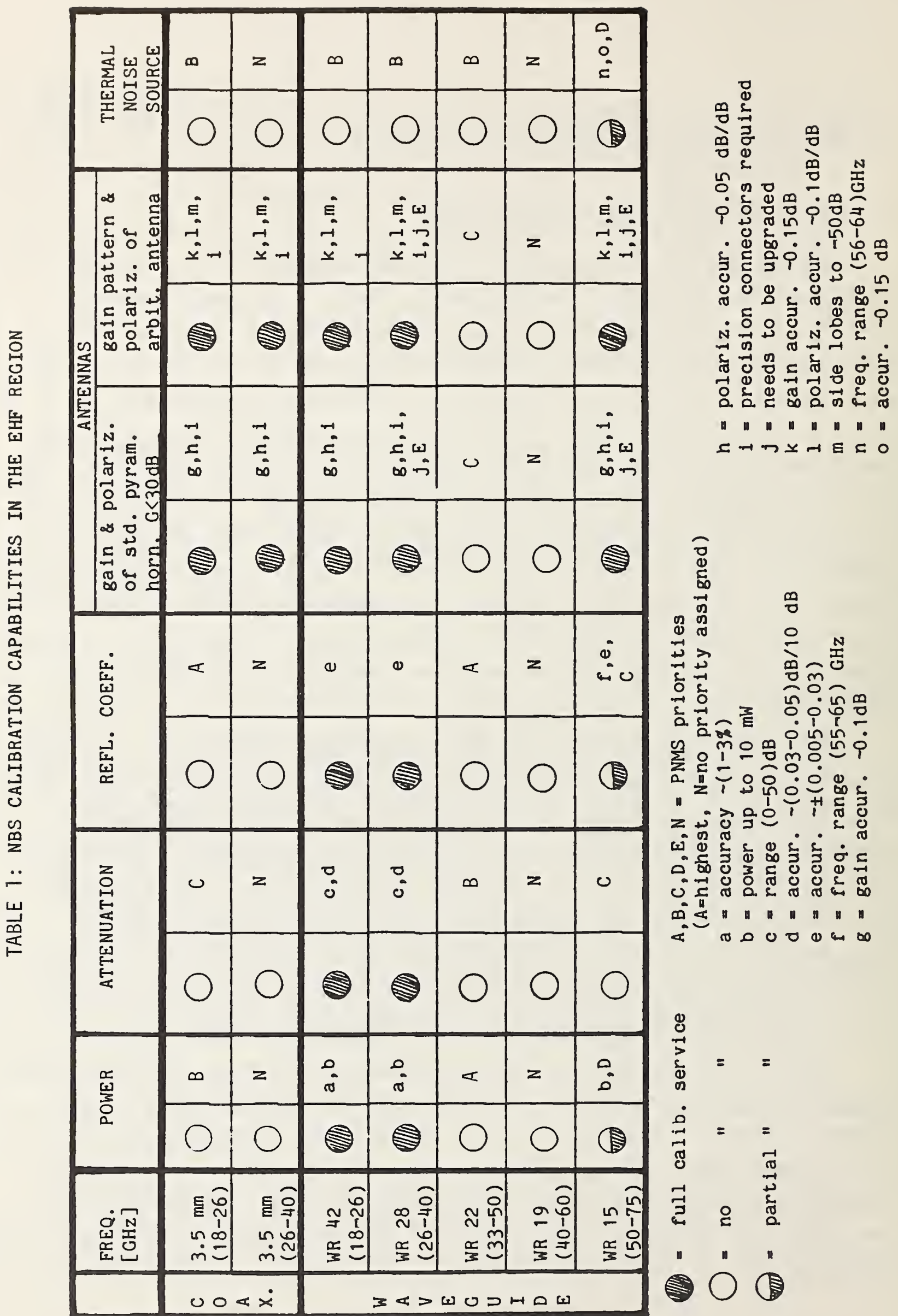


7. Conclusions and Recommendations

For SHF/EHF satellite systems (MILSTAR), it will be the primary function of the AFSCF test facilities to provide the on-orbit calibration of the communications payloads. To meet these requirements, test terminals must be sized to provide calibration capabilities for the EHF uplink and SHF downlink parameters.

A number of testing and calibration requirements for such systems have been examined in this study. For example, in the on-board power detection system, the error in the uplink received isotropic power measurement is bounded by $0.2 \mathrm{~dB}$. This will require antenna gain accuracies and power meter accuracies to be well within $\pm 0.15 \mathrm{~dB}$ since error uncertainties in attenuator, reflection coefficients, connectors, etc. are also contributing factors to the total error. Compliance with this requirement will be difficult, if not unattainable. In light of this example, the test facility must also have the capability to measure not only the EHF uplink EIRP parameters and SHF downlink G/T parameters within stringent error bounds, but also various parameters for the satellite transmit and receive parameters including polarization, axial ratio, beamwidth, beampointing, antenna coverage, bit error rates, etc. within strict error bounds.

These requirements appear to be based largely on earlier satellite systems (such as DSCS) which are less complex and also operate at lower frequencies. These two factors have profound effects on measurements and calibrations at EHF/SHF frequencies in that the achievable accuracies are yet to be determined for individual parameter measurements. Some of these measurements are greatly affected by the atmosphere whose state has not yet been established accurately.

Two principal reasons for embarking on a program supporting calibrations and measurements are: first, to ensure the continued operational health of the system during its life and to be able to identify modifications and repairs which permit operation in the event of degraded performance or failure; and second, to establish the level of satellite performance that permits compliance with incentive payments to the satellite vendor. Because of the considerable difficulty and expense associated with measurements at and beyond the state-of-the-art, a careful trade-off analysis should be conducted between stated requirements and the commitments needed to achieve that level of 
capability. For example, if measurement of the uplink behavior of the system must be known very accurately to adequately monitor system performance, then weight, power, and cost must be assigned out of the spacecraft budgets during the satellite design phase in order to meet this goal. If the uncertainty of measurement must indeed be no larger than $0.2 \mathrm{~dB}$, then three steps should be undertaken. First, system testing must be given a priority on allocation of system time, so that relatively rare opportunities of ideal meteorological conditions can be utilized for test purposes by preemption. Second, a program of atmospheric research should be initiated so as to be able to precisely identify atmospheric states and to make loss corrections of sufficient accuracy to meet the requirement. Third, calibration techniques and standards at SHF/EHF frequencies for the specific parameters need to be evaluated and/or developed to assure the highest accuracy, or, equivalently, the lowest error bounds.

Recognizing that these recommendations are in themselves difficult to implement at the present stage of systems development, we are including a suggested series of efforts that need to be accomplished in order to establish the measurement and calibration capabilities required to support AFSCF and Camp Parks in their mission.

Atmospheric and Noise Requirements

1. Development of laboratory noise calibration services in the waveguide sizes required to support the 20 and $44 \mathrm{GHz}$ frequency bands

Table 1 of this report shows that there has been to date sufficiently little demand for these services that NBS resources have been directed elsewhere. The MILSTAR application identifies a single requirement for this service, and therefore does not justify using general purpose funding to the detriment of broader national programs now underway.

2. Accurate characterization of the atmosphere

To measure EIRP from a satellite at EHF frequencies, the atmospheric loss must be known. The atmosphere will cause measurement errors of about $0.5 \mathrm{~dB}$ for ideal conditions and errors up to the order of $3 \mathrm{~dB}$ for cloudy or rainy conditions. More precise atmospheric models and adequate measurement methods need to be developed. 
3. Accurate characterization of Sun and Moon at EHF

If the noise flux from extraterrestrial sources is well known, these sources can be used as far-field test standards for calibrating Earth terminals. They are particularly attractive for use in remote locations where it may not be feasible to transport calibrated antennas and other standards. Moreover, calibrated extraterrestrial sources can be used effectively to evaluate and develop improved atmospheric loss models. Since the Sun and Moon are potentially the most useful sources at EHF frequencies, they must be accurately measured and characterized.

4. Techniques for evaluation of connectors and adaptors

Measurement and calibration techniques developed in a primary standards laboratory use the highest quality connectors available in order to achieve the lowest levels of uncertainties in the results. Such connectors are not usually used in field and space applications because of fragility, size, cost, or other considerations. Therefore, a calibration performed in the standards laboratory must be physically transferred to the field environinent through appropriate adaptors and connectors. In the EHF region, this can be accompanied by severe degradation in accuracy unless techniques are available for identifying and correcting for the imperfections in these devices. This is an area in which comparatively little research has been done [19], but under the combined pressure of greater degradation at these frequencies and more stringent requirements for advanced systems, such work is needed.

\section{Antenna Calibration Requirements}

1. Upgrade and supplement laboratory equipment for $44 \mathrm{GHz}$ antenna calibrations

As with noise calibration, there has been no specific demand for antenna measurement services at this frequency. Thus, NBS is not properly equipped to do these calibrations without specific support. Among other prerequisites, this means that the presently available range of attenuator calibrations must be extended to cover a dynamic range of at least $40 \mathrm{~dB}$. 
2. Develop modifications in extrapolation and near-field antenna calibration techniques that avoid cable flexing problems

Highly accurate antenna calibrations at EHF are thrusting against the limits of basic technology. The near-field and extrapolation techniques developed at NBS for accurate antenna gain measurements require considerable physical movement of the antenna under test while it is connected into the system. Therefore, cables whose attenuation and phase transfer characteristics are unstable become limiting factors in the accuracy of the resulting calibration. Present capability is adequate up to about $30 \mathrm{GHz}$, but the cable problem will have to be resolved in order to meet the requirements at $44 \mathrm{GHz}$.

3. Develop swept frequency antenna gain measurement techniques

This is needed because calibrations are not normally performed at exact operating frequencies but at fixed points with a swept frequency calibration to aid interpolation. In order to retain high accuracy, new equipment and revised techniques are needed.

4. Develop CP antenna gain and polarization standards and associated probes

Most present antenna calibrations are done with linearly polarized fields. Although circularly polarized antennas can be handled to a limited degree with these techniques, maximum attainable accuracy and efficiency of calibration requires a modification of technique to operate directly in circular polarization. This in turn requires antennas designed and constructed for this purpose, rather than simply using available antennas which have been developed for other applications.

5. Perform error analysis on gain transfer calibration techniques, including effect of imperfections in standard antennas

Gain transfer measurement techniques for antennas are likely to play an important part in support of the MILSTAR system. Because of the difficulty of calibrating the gain of all MILSTAR Earth station antennas, it is likely that gain standards will be calibrated at NBS and these used to transfer gain measurements to CPCA and to other sites. Moreover, having a gain standard on site can provide an additional means of evaluating atmospheric loss in real time. 


\section{References}

[1] Bhargava, V. K.; Haccoun, D.; Matyas, R.; Nuspl, P. Digital communications by satellite. New York: John Wiley \& Sons; 1981.

[2] Kreutal, R. W., Jr.; DiFonso, D. F.; Mahle, C. E. Satellite system measurements. Proc. IEEE 66(4): 472; 1978 April.

[3] Ippolito, L. J.; Kaul, R. D.; Wallace, R. G. Propagation effects handbook for satellite systems design. NASA Ref. Pub. 1082(3); 1983 June.

[4] Yokoi, H.; Yamada, M.; Satak, T. Atmospheric attenuation and scintilation of microwaves from outer space. Publ. Astr. Soc. Japan 22(4): 511-524; 1970.

[5] Yamada, M.; Yokoi, H. Measurements of Earth-space propagation characteristics at $15.5 \mathrm{Ghz}$ and $31.6 \mathrm{GHz}$ using celestial radio sources. J. of the Inst. of Electronics and Communications Engineers of Japan 57$\mathrm{B}(2) ; 1974$ February.

[6] Wait, D. F.; Daywitt, W. C.; Kanda, M.; Miller, C. K. S. A study of the measurement of G/T using Cassiopeia A. Nat. Bur. Stand. (U.S.) NBSIR 74$382 ; 1974$ June.

[7] Wait, D. F. Error predictions for 1-60 GHz G/T measurements using celestial radio sources (to be published).

[8] Daywitt, W. C. 10-60 GHz G/T measurements using the Sun as a source. Nat. Bur. Stand. (U.S.) NBSIR 86-3046; April 1986.

[9] Falcone, V. J., Jr.; Wulfberg, K. N.; Gitelson, S. Atmospheric emission and absorption at millimeter wavelengths. Radio Science 6(3): 347; 1971 March.

[10] Newell, A. C.; Crawford, M. L. Planar near-field measurements on high performance array antennas. Nat. Bur. Stand. (U.S.) NBSIR 74-380; 1974 July.

[11] Yaghjian, A. D. Near-field measurements on a cylindrical surface: A source scattering-matrix formulation. Nat. Bur. Stand. (U.S.) Tech. Note 696; 1977 September.

[12] Wacker, P. F. Nonplanar near-field measurements: Spherical scanning. Nat. Bur. Stand. (U.S.) NBSIR 75-809; 1975 June.

[13] Newel 1, A. C.; Baird, R. C.; Wacker, P. F. Accurate measurement of antenna gain and polarization at reduced distances by an extrapolation technique. IEEE Trans. Antennas Propag. AP-21: 418-431; 1973.

[14] Newell, A. C.; Kerns, D. M. Determination of both polarization and power gain of antennas by a generalized 3-antenna measurement method. Electron. Lett. 7: 68-70; 1971 February 11. 
[15] Newell, A. C. Improved polarization measurements using a modified three antenna technique. Session 15, 1975 June 2-4; Urbana/Champaign, Il. Proc. IEEE Int. Antennas and Propagation Symp.; 1975. 337-340.

[16] Johnson, R. C.; Ecker, H. A.; Moore, R. A. Compact range techniques and measurements. IEEE Trans. Antennas Propag. AP-17: 568-576; 1969 September.

[17] Repjar, A. G.; Kremer, D. P. Accurate evaluation of a millimeter wave compact range using planar near-field scanning. IEEE Trans. Antennas Propag. AP-30(3); 1982 May.

[18] Kanda, M. Accuracy considerations in the measurement of the power gain of a large microwave antenna. IEEE Trans. Antennas Propag. AP-23(3): 407-411; May 1975.

[19] Estin, A. J. Scattering pararneters of SMA connector pairs. IEEE Trans. Instrum. Meas. IM-25(4): 329; 1976 December. 


\begin{tabular}{|c|c|c|c|}
\hline $\begin{array}{c}\text { U.S. OEPT. OF COMM. } \\
\text { BIBLIOGRAPHIO DATA } \\
\text { SHEET (See instructions) }\end{array}$ & $\begin{array}{c}\text { 1. PUBLICATION OR } \\
\text { REPORT NO. } \\
\text { NBSIR 86-3058 }\end{array}$ & 2. Performing Organ. Report No. & $\begin{array}{c}\text { 3. Publication Date } \\
\text { October } 1986\end{array}$ \\
\hline
\end{tabular}

4. TITLE AND SUBTITLE

Calibration Requirements for EHF Satellite Communication Systems

5. AUTHOR(S)

R. C. Baird, W. C. Daywitt, A. C. Newel1, S. Perera, A. G. Repjar, D. F. Wait, A.J. Estitn 6. PERFORMING ORGANIZATION (If joint or other than NBS, see instructions)

NATIONAL BUREAU OF STANDARDS

DEPARTMENT OF COMMERCE

7. Contract/Grant No.

WASHINGTON, D.C. 20234

9. SPONSORING ORGANIZATIOH ISAME AND COMPLETE ADDRESS (SIfEEt, City, State, ZIF)

8. Type of Report \& Period Covered

Air Force Satellite Control Facility

Sunnyvale, Cal ifornia 94088

10. SUPPLEMENTARY NOTES

Document describes a computer program; SF-185, FIPS Software Summary, is attached.

11. ABSTRACT (A 200-word or less factual summary of most significant information. If document includes a significant bibliography or literature survey, mention it here)

The calibration and measurement support requirements of millimeter wave satellite systems such as MILSTAR have been investigated. The needs for measurements on satellite sy'stems are reviewed. An overview of the various means available for calibrating antenna gain, one of the key measurements that needs to be accurately accomplished, is presented. Essentially three new measurement problems arise because of operating in the upper SHF and EHF frequency ranges. First, without adequate methods to measure the atmospheric loss, the accuracy of effective isotropic radiated power (EIRP) measurements in the $20-45 \mathrm{GHz}$ range can be no better than $0.5-3 \mathrm{~dB}$ (depending on frequency and antenna elevation angle), which is inadequate for MILSTAR requirements. Second, standards and measurement support services are not presently available from the National Bureau of Standards and are needed to support millimeter wave antenna gain and thermal noise measurements. Third, if the Sun and/or Moon are to be used for measuring Earth terminal G/T, Earth terminal antenna gain, or satellite EIRP in the millimeter region, they need to be appropriately characterized at those frequencies.

12. KEY WORDS (Six to twelve entries; alphabetical order: capitalize only proper names; and separate key words ty semicolons) antenna gain calibration; atmospheric loss; effective isotropic radiated power; G/T; MILSTAR; satellite communications; solar noise flux

13. AVAILABILITY

[X] Unlimited

$\square$ For Official Distribution. Do Not Release to NTIS

Order From Superintendent of Documents, U.S. Government Printing Office, Washington, D.C. 20402.

14. NO. OF PRINTED PAGES

X] Order From National Technical Information Service (NTIS), Springfield, VA. 22161 


